\title{
AN INTERPOLATION SERIES EXPANSION FOR A MEROMORPHIC FUNCTION
}

\author{
BY \\ J. L. WALSH
}

A function $f(z)$ meromorphic at every finite point of the plane can be expanded $\left({ }^{1}\right)$ in a suitable interpolation series

$$
\begin{aligned}
f(z) \equiv \frac{a_{1}}{z-\alpha_{1}} & +a_{2} \frac{z-\beta_{1}}{\left(z-\alpha_{1}\right)\left(z-\alpha_{2}\right)} \\
& +a_{3} \frac{\left(z-\beta_{1}\right)\left(z-\beta_{2}\right)}{\left(z-\alpha_{1}\right)\left(z-\alpha_{2}\right)\left(z-\alpha_{3}\right)}+\cdots,
\end{aligned}
$$

where all the poles of $f(z)$ occur among the points $\alpha_{k}$, where the coefficients $a_{k}$ are found by formal interpolation in the points $\beta_{k}$, and where the expansion (1) is valid for every $z\left(\neq \alpha_{k}\right)$. The purpose of the present note is to indicate that a function meromorphic merely in the deleted neighborhood of a point $O$ (it is sufficient if the function has at $O$ an essential singularity of the second kind) also admits an expansion of form (1) valid in a neighborhood of $O$. Our primary method is the use of uniformly distributed points, which (in a special case of polynomials) were first used for interpolation by Hilbert and Fejér. But the present use of such points involves certain novelties, and is by no means an immediate application of hitherto existing methods.

THEOREM 1. Let the Jordan curve $C_{0}$ contain in its interior the Jordan curve $C_{1}$ and the origin $O$, with $O$ interior to $C_{1}$, and let the sequence of points $\alpha_{1}^{\prime}, \alpha_{2}^{\prime}, \cdots$ be disjoint from $C_{1}$ and approach $O$. Then there exist points $\beta_{1}, \beta_{2}, \cdots$ on $C_{1}$ and a sequence $\alpha_{1}, \alpha_{2}, \cdots$ of which $\alpha_{1}^{\prime}, \alpha_{2}^{\prime}, \cdots$ is a subsequence and the remaining points $\alpha_{k}$ lie on $C_{0}$, with the following property: every function $f(z)$ analytic in the closed interior of $C_{1}$, except perhaps at $O$ and except perhaps for poles in the points $\alpha_{k}^{\prime}$, admits an expansion (1) found formally by interpolation in the points $\beta_{k}$ and valid uniformly on any closed set in the closed interior of $C_{1}$ but not containing $O$ nor any of the points $\alpha_{k}$.

Let $U(z)$ be the function harmonic in the annulus $A$ bounded by $C_{0}$ and $C_{1}$, continuous in the corresponding closed region, equal to zero on $C_{0}$ and to unity on $C_{1}$. If the points $\alpha_{k}^{\prime \prime}$ are uniformly distributed on $C_{0}$ and the points $\beta_{k}^{\prime \prime}$ on $C_{1}$ in the sense of Weyl with respect to the conjugate of $U(z)$, then we have (op. cit. $\S \S 8.7$ and 8.8)

Received by the editors March 14, 1952.

(1) J. L. Walsh, Interpolation and approximation, Amer. Math. Soc. Colloquium Publications, vol. 20, 1935, p. 222 (Corollary 2). 
(2) $\lim _{n \rightarrow \infty}\left|\frac{\left(z-\beta_{1}^{\prime \prime}\right)\left(z-\beta_{2}^{\prime \prime}\right) \cdots\left(z-\beta_{n}^{\prime \prime}\right)}{\left(z-\alpha_{1}^{\prime \prime}\right)\left(z-\alpha_{2}^{\prime \prime}\right) \cdots\left(z-\alpha_{n}^{\prime \prime}\right)}\right|^{1 / n}=\left\{\begin{array}{l}\exp [\tau U(z)], z \text { in } A, \\ \exp \tau, z \text { interior to } C_{1},\end{array}\right.$

where $\tau$ is a suitably chosen negative constant, and where the respective limits are approached uniformly on any closed set in $A$ and on any closed set interior to $C_{1}$. We notice that a consequence of (2) is

$$
\lim _{n \rightarrow \infty}\left[\max \left|\frac{\left(z-\beta_{1}^{\prime \prime}\right) \cdots\left(z-\beta_{n}^{\prime \prime}\right)}{\left(z-\alpha_{1}^{\prime \prime}\right) \cdots\left(z-\alpha_{n}^{\prime \prime}\right)}\right|, z \text { on } C_{1}\right]^{1 / n}=e^{\tau} .
$$

Indeed, this rational function of $z$ is analytic throughout the interior of $C_{0}$; its maximum modulus on $C_{1}$ is not greater than its maximum modulus on the curve $U(z)=\sigma$ in $A$, so the first member of (3) is not greater than $e^{\sigma \tau}$ for every $\sigma, 0<\sigma<1$, hence is not greater than $e^{\tau}$. If the first member of (3) were less than $e^{r}$, equation (2) for $z$ interior to $C_{1}$ would not be valid.

Moreover, if the points $\beta_{k}^{\prime}$ are uniformly distributed on $C_{1}$ with respect to the conjugate of $g(z)$, Green's function for the interior $R_{1}$ of $C_{1}$ with pole in $O$, then we have (op. cit., pp. 214-215)

$$
\begin{aligned}
\lim _{n \rightarrow \infty}\left|\frac{\left(z-\beta_{1}^{\prime}\right)\left(z-\beta_{2}^{\prime}\right) \cdots\left(z-\beta_{n}^{\prime}\right)}{z^{n}}\right|^{1 / n} & \left\{\begin{array}{cl}
\exp [g(z)], & z(\neq 0) \text { interior to } R_{1}, \\
1, & z \text { exterior to } C_{1},
\end{array}\right.
\end{aligned}
$$

and the limits are approached uniformly on any closed sets in $R_{1}-0$ and exterior to $C_{1}$ respectively. As in the proof of (3) we have

$$
\lim _{n \rightarrow \infty}\left[\max \left|\frac{\left(z-\beta_{1}^{\prime}\right) \cdots\left(z-\beta_{n}^{\prime}\right)}{z^{n}}\right|, z \text { on } S\right]^{1 / n}=e^{r},
$$

where $S$ is any closed annulus whose interior boundary is the curve $g(z)=r$ interior to $C_{1}$.

The relation $\alpha_{k}^{\prime} \rightarrow 0$ implies log $\left|z-\alpha_{k}^{\prime}\right| \rightarrow \log |z|$ and hence (by Cesàro summation) implies $\log \left|\left(z-\alpha_{1}^{\prime}\right) \cdots\left(z-\alpha_{n}^{\prime}\right)\right|^{1 / n} \rightarrow \log |z|$, whence $\left(z \neq \alpha_{k}^{\prime}\right)$

$$
\lim _{n \rightarrow \infty}\left|\frac{\left(z-\beta_{1}^{\prime}\right) \cdots\left(z-\beta_{n}^{\prime}\right)}{z^{n}}\right|^{1 / n}=\lim _{n \rightarrow \infty}\left|\frac{\left(z-\beta_{1}^{\prime}\right) \cdots\left(z-\beta_{n}^{\prime}\right)}{\left(z-\alpha_{1}^{\prime}\right) \cdots\left(z-\alpha_{n}^{\prime}\right)}\right|^{1 / n},
$$

provided the first member exists; and uniformity in the first member implies uniformity in the second member on any closed set not containing $O$ or a point $\alpha_{k}^{\prime}$. Equation (6) is to be used in conjunction with (4) and (5).

The points $\beta_{k}$ of Theorem 1 are to be obtained from the sets of points $\beta_{k}^{\prime}$ and $\beta_{k}^{\prime}$, and the points $\alpha_{k}$ are to be obtained from the sets $\alpha_{k}^{\prime}$ and $\alpha_{k}^{\prime \prime}$. 
The precise choice of the $\beta_{k}$ and $\alpha_{k}$ requires further discussion.

Let $C$ denote the fixed Jordan curve $|g(z)|=\rho(>0)$ interior to $C_{1}$, and let the sequence of numbers $B_{n}$ be chosen to become infinite monotonically, with

$$
B_{n} \geqq\left|\frac{\left(z-\beta_{1}^{\prime \prime}\right) \cdots\left(z-\beta_{n}^{\prime \prime}\right)\left(t-\alpha_{1}^{\prime \prime}\right) \cdots\left(t-\alpha_{n}^{\prime \prime}\right)}{\left(z-\alpha_{1}^{\prime \prime}\right) \cdots\left(z-\alpha_{n}^{\prime \prime}\right)\left(t-\beta_{1}^{\prime \prime}\right) \cdots\left(t-\beta_{n}^{\prime \prime}\right)}\right|
$$

uniformly for $z$ on $C_{1}$ and $t$ on $C$, hence uniformly for $z$ in the closed interior of $C_{1}$ and $t$ in the closed interior of $C$. We suppose also

$$
\lim _{n \rightarrow \infty} B_{n}^{1 / n}=1 \text {; }
$$

it will be noted that by (2) and (3) the $n$th root of the maximum of the second member of (7) approaches unity uniformly for $z$ on $C_{1}$ and $t$ on $C$, so such a sequence $B_{n}$ exists.

We choose $M_{n}(<n)$ as a sequence of positive integers with

$$
M_{n} \leqq M_{n+1} \leqq M_{n}+1
$$

and

$$
\lim _{n \rightarrow \infty} \frac{M_{n} \rho}{2 \log B_{n}}=1
$$

From (8) we have then

$$
\lim _{n \rightarrow \infty} \frac{\log B_{n}}{n}=0, \quad \lim _{n \rightarrow \infty} \frac{M_{n}}{n}=0 .
$$

We define the monotonic sequence of integers $N_{n}=n-M_{n}>0$, whence $N_{n} / n \rightarrow 1$, and shall prove that the relation

$$
\limsup _{n \rightarrow \infty} A_{n}^{1 / M_{n}} \leqq e^{-\infty}<1
$$

implies

$$
\lim _{n \rightarrow \infty}\left(A_{n} B_{n}\right)=0 .
$$

We have for $n$ sufficiently large by (9) and (11)

$$
\log \left(A_{n} B_{n}\right)=\log B_{n}-\log B_{n} \frac{M_{n} \rho}{\log B_{n}} \frac{-\log A_{n}}{M_{n} \rho}<-\frac{1}{2} \log B_{n} \rightarrow-\infty,
$$

which implies (12).

The formal development (1) found by interpolation in the points $\beta_{k}$ is equivalent (op cit., p. 190) to the formal development 


$$
\left[\frac{\left(z-\alpha_{1}\right) \cdots\left(z-\alpha_{m}\right)}{\left(z-\beta_{1}\right) \cdots\left(z-\beta_{m}\right)}\right]\left[f(z)-\frac{a_{1}}{z-\alpha_{1}}-\cdots\right.
$$

$$
\begin{array}{r}
\left.-a_{m} \frac{\left(z-\beta_{1}\right) \cdots\left(z-\beta_{m-1}\right)}{\left(z-\alpha_{1}\right) \cdots\left(z-\alpha_{m}\right)}\right] \\
=\frac{a_{m+1}}{z-\alpha_{m+1}}+a_{m+2} \frac{z-\beta_{m+1}}{\left(z-\alpha_{m+1}\right)\left(z-\alpha_{m+2}\right)}+\cdots,
\end{array}
$$

again a series of type (1). The advantage of (13) as compared with (1) is twofold: that (13) is the expansion of a function $f_{1}(z)$ which may be analytic in the points $\alpha_{1}, \alpha_{2}, \cdots, \alpha_{m}$ even though $f(z)$ has poles in those points, and also that the poles $\alpha_{k}(k \leqq m)$ no longer appear in the second member of (13).

We now define the points $\alpha_{k}$ by the requirement that the set $\alpha_{1}, \alpha_{2}, \cdots, \alpha_{n}$ shall contain each of the sets $\alpha_{1}^{\prime}, \alpha_{2}^{\prime}, \cdots, \alpha_{M_{n}}^{\prime}$ and $\alpha_{1}^{\prime \prime}, a_{2}^{\prime \prime}, \cdots, \alpha_{N_{n}}^{\prime \prime}$ without inversion of order, and define the points $\beta_{k}$ by the similar requirement that the set $\beta_{1}, \beta_{2}, \cdots, \beta_{n}$ shall contain each of the sets $\beta_{1}^{\prime}, \beta_{2}^{\prime}, \cdots, \beta_{M_{n}}^{\prime}$ and $\beta_{1}^{\prime \prime}, \beta_{2}^{\prime \prime}, \cdots, \beta_{N_{n}}^{\prime \prime}$ without inversion of order.

The validity of (1) or (13) is a consequence of the interpolation formula (op. cit., p. 186)

$$
\begin{aligned}
& f_{1}(z)-r_{n}(z) \\
& \quad \equiv \frac{1}{2 \pi i} \int_{\Gamma+\gamma} \frac{\left(z-\beta_{1}\right) \cdots\left(z-\beta_{n}\right)\left(t-\alpha_{1}\right) \cdots\left(t-\alpha_{n}\right) f_{1}(t) d t}{\left(z-\alpha_{1}\right) \cdots\left(z-\alpha_{n}\right)\left(t-\beta_{1}\right) \cdots\left(t-\beta_{n}\right)(t-z)} ;
\end{aligned}
$$

here $r_{n}(z)$ denotes the sum of the first $n$ terms of series (1); we suppose $f_{1}(z)$ analytic in the closed annular region bounded by $\Gamma$ : a curve $U(z)=\sigma$ in $A$, and $\gamma:$ a curve $g(z)=$ const. interior to $C_{1}$; the equation is valid for $z$ lying between $\Gamma$ and $\gamma$. We have expressed (14) in terms of $f_{1}(z)$ rather than $f(z)$ to secure the advantages of (13) already mentioned. We note that the relations (2) and (4) remain valid if a finite number of factors in numerator and denominator are omitted.

Let $S$ be an arbitrary closed set in the closed interior of $C_{1}$ containing none of the points 0 or $\alpha_{k}^{\prime}$. Let $S$ lie exterior to the curve $g(z)=r$; we choose $\gamma$ as the curve $g(z)=r+\rho$, so that $\gamma$ lies interior to the curve $C$ previously introduced. We estimate the second member of (14) by writing the kernel in the form

$$
\begin{aligned}
& \frac{\left(z-\beta_{1}\right) \cdots\left(z-\beta_{n}\right)\left(t-\alpha_{1}\right) \cdots\left(t-\alpha_{n}\right)}{\left(z-\alpha_{1}\right) \cdots\left(z-\alpha_{n}\right)\left(t-\beta_{1}\right) \cdots\left(t-\beta_{n}\right)} \\
& \quad=\frac{\left(z-\beta_{1}^{\prime}\right) \cdots\left(z-\beta_{M_{n}}^{\prime}\right)\left(t-\alpha_{1}^{\prime}\right) \cdots\left(t-\alpha_{M_{n}}^{\prime}\right)\left(z-\beta_{1}^{\prime \prime}\right) \cdots\left(z-\beta_{N_{n}}^{\prime \prime}\right)\left(t-\alpha_{1}^{\prime \prime}\right) \cdots\left(t-\alpha_{N_{n}}^{\prime \prime}\right)}{\left(z-\alpha_{1}^{\prime}\right) \cdots\left(z-\alpha_{M_{n}}^{\prime}\right)\left(t-\beta_{1}^{\prime}\right) \cdots\left(t-\beta_{M_{n}}^{\prime}\right)\left(z-\alpha_{1}^{\prime \prime}\right) \cdots\left(z-\alpha_{N_{n}}^{\prime \prime}\right)\left(t-\beta_{1}^{\prime \prime}\right) \cdots\left(t-\beta_{N_{n}}^{\prime \prime}\right)}
\end{aligned}
$$

When $z$ lies on $S$ and $t$ lies on $\Gamma$, the second member of (15) can be expressed as four factors the superior limits of whose $n$th roots are respectively not 
greater than

$$
1,1, e^{\tau}, e^{-\sigma \tau}
$$

consequently the second member of (15), and hence the second member of (14) where the integral is taken over $\Gamma$, approaches zero. When $z$ lies on $S$ and $t$ lies on $\gamma$, the second member of (15) is to be compared to (7) and (14); by the monotonic character of the $B_{n}$ and (7), the second member of (15) is not greater than $A_{n} \cdot B_{n}$, where

$$
A_{n}=\max \left|\frac{\left(z-\beta_{1}^{\prime}\right) \cdots\left(z-\beta_{M_{n}}^{\prime}\right)\left(t-\alpha_{1}^{\prime}\right) \cdots\left(t-\alpha_{M_{n}}^{\prime}\right)}{\left(z-\alpha_{1}^{\prime}\right) \cdots\left(z-\alpha_{M_{n}^{\prime}}^{\prime}\right)\left(t-\beta_{1}^{\prime}\right) \cdots\left(t-\beta_{M_{n}^{\prime}}\right)}\right|,
$$

for $z$ on $S$ and $t$ on $\gamma$. From (4), (5), and (6) we have

$$
\limsup _{n \rightarrow \infty} A_{n}^{1 / M_{n}} \leqq e^{-\rho}<1
$$

and it follows from (12) that the second member of (14) approaches zero, uniformly for $z$ on $S$ and $t$ on $\gamma$. Thus $r_{n}(z)$ approaches zero uniformly for $z$ on $S$.

The notation of (14) is not completely descriptive, for the points $\alpha_{k}$ and $\beta_{k}$ in (14) are those of (1) rather than of (13). However, we have proved that as $n$ becomes infinite the kernel of (14) approaches zero uniformly for $z$ on $S$ and for $t$ on $\Gamma$ or $\gamma$. This property holds even if a finite number of factors of the kernel are omitted, and therefore holds for the kernel associated with (13). Theorem 1 is established.

Theorem 1 includes the case that $f(z)$ is analytic in $R_{1}-O+C_{1}$, hence includes the case that $f(z)$, analytic in $R_{1}-O+C_{1}$, is analytic or has either a pole or essential singularity of the first kind in $O$; here the points $\alpha_{k}^{\prime}$ may all coincide at $O$.

For the sake of simplicity we have formulated Theorem 1 in the case of a region bounded by a single Jordan curve. Conclusion and proof are valid if $C_{0}$ and $C_{1}$ each consists of a finite number of mutually disjoint Jordan curves, with $C_{0}$ the boundary of a region $R_{0}$ containing $C_{1}$, and with $C_{1}$ the boundary of a subregion containing 0 .

In Theorem 1 we have not proved the convergence of the sequence $r_{n}(z)$ to $f(z)$ at points of $A$; we now show by means of an example that such convergence cannot be proved without change of hypothesis or method. The sequence $M_{n}$ does not depend on the $\alpha_{k}^{\prime}$, so we define a particular set of $\alpha_{k}^{\prime}$ in terms of the $M_{n}$. For each $k(>0)$ let $n(k)$ be the smallest $n$ for which we have $M_{n}=k$; we set

$$
\lambda_{k}=\frac{k}{n(k)}=\frac{M_{n(k)}}{n(k)},
$$


which approaches zero. We also have $\lambda_{k} \log \left(-\log \lambda_{k}\right) \rightarrow 0$. For each $n$ we have

$$
\lambda_{M_{n}}=\frac{M_{n}}{n\left(M_{n}\right)} \geqq \frac{M_{n}}{n},
$$

whence

$$
\lim _{n \rightarrow \infty} \frac{M_{n}}{n} \log \left(-\log \lambda_{M_{n}}\right)=0 .
$$

We now introduce the definition for $k$ sufficiently large $\alpha_{k}^{\prime}=\alpha_{1}^{\prime} /\left(-\log \lambda_{k}\right)$, where $\alpha_{1}^{\prime}$ interior to $C_{1}$ and different from 0 is arbitrary, and where the $\alpha_{k}^{\prime}$ not defined are chosen arbitrarily interior to $C_{1}$ but different from zero; of course $\alpha_{k}^{\prime} \rightarrow 0$. For $n$ sufficiently large we have

$$
\begin{aligned}
& \left|\alpha_{1}^{\prime} \alpha_{2}^{\prime} \cdots \alpha_{M_{n}}^{\prime}\right|^{1 / n} \geqq\left|\frac{\alpha_{1}^{\prime}}{-\log \lambda_{M_{n}}}\right|^{M_{n} / n}, \\
& \log \left|\frac{\alpha_{1}^{\prime}}{-\log \lambda_{M_{n}}}\right|^{M_{n} / n}=\frac{M_{n}}{n}\left[\log \left|\alpha_{1}^{\prime}\right|-\log \left(-\log \lambda_{M_{n}}\right)\right] \rightarrow 0 .
\end{aligned}
$$

For the particular function $f(z) \equiv 1 / z$ we have (op. cit., p. 185)

(18) $f(z)-r_{n}(z)=\frac{\left(z-\beta_{1}^{\prime}\right) \cdots\left(z-\beta_{M_{n}}^{\prime}\right)\left(-\alpha_{1}^{\prime}\right) \cdots\left(-\alpha_{M_{n}}^{\prime}\right)\left(z-\beta_{1}^{\prime \prime}\right) \cdots\left(z-\beta_{N_{n}}^{\prime \prime}\right)\left(-\alpha_{1}^{\prime \prime}\right) \cdots\left(-\alpha_{N_{n}}^{\prime \prime}\right)}{\left(z-\alpha_{1}^{\prime}\right) \cdots\left(z-\alpha_{M_{n}}^{\prime}\right)\left(-\beta_{1}^{\prime}\right) \cdots\left(-\beta_{M_{n}}^{\prime}\right)\left(z-\alpha_{1}^{\prime \prime}\right) \cdots\left(z-\alpha_{N_{n}}^{\prime \prime}\right)\left(-\beta_{1}^{\prime \prime}\right) \cdots\left(-\beta_{N_{n}}^{\prime \prime}\right)}$.

Let $z$ be an arbitrary point of $A$ with $U(z)=\sigma$. The modulus of the $n$th root of the second member of (18) is studied in four distinct factors by means of (6), (4), (16), (17), (2), and is seen to have an inferior limit not less than

$$
1 \cdot 1 \cdot e^{\sigma \tau} \cdot e^{-\tau}=e^{(\sigma-1) r}>1,
$$

so the sequence $r_{n}(z)$ does not converge.

Theorem 1 is the study of a series of interpolation (1) rather than a study of a more general sequence of rational functions with assigned poles and determined by interpolation in assigned points, but the latter study is indeed simpler and can be treated by similar methods to yield similar results. For simplicity we limit ourselves to a special situation:

THEOREM 2. Let $C_{0}$ and $C_{1}$ be the respective circles $|z|=1$ and $|z|=r_{1}$, with $1>r_{1}$, and let the sequence $\alpha_{1}^{\prime}, \alpha_{2}^{\prime}, \cdots$ be disjoint from $C_{1}$ and approach 0. Let $\left\{M_{n}\right\}$ be a monotonic nondecreasing unbounded sequence of integers with $M_{n+1} \leqq M_{n}+1$ and $\lim _{n \rightarrow \infty} M_{n} / n=0$; we set $N_{n}=n-M_{n}$. For each $n$ let the points $\alpha_{n 1}, \alpha_{n 2}, \cdots, \alpha_{n n}$ consist of the points $\alpha_{1}^{\prime}, \alpha_{2}^{\prime}, \cdots, \alpha_{M_{n}}^{\prime}$ and all the $N_{n}$ th roots of unity, and let the points $\beta_{n 1}, \beta_{n 2}, \cdots, \beta_{n n}$ consist of the $M_{n}$ th roots of $r_{1}^{M_{n}}$ and the $N_{n}$ th roots of $r_{1}^{N_{n}}$. Let $r_{n}(z)$ denote the rational function of form 


$$
r_{n}(z)=\frac{A_{n 1} z^{n-1}+A_{n 2} z^{n-2}+\cdots+A_{n n}}{\left(z-\alpha_{n 1}\right) \cdots\left(z-\alpha_{n n}\right)}
$$

defined by interpolation to $f(z)$ in the points $\beta_{n k}$, where $f(z)$ is an arbitrary function analytic in the closed interior of $C_{1}$ with 0 deleted, except perhaps for poles all of which lie in the points $\alpha_{k}^{\prime}$. Then the sequence $r_{n}(z)$ converges uniformly to $f(z)$ on any closed set $S$ in the closed interior of $C_{1}$ not containing 0 nor a point $\alpha_{k}$.

Here equation (14) is replaced by a similar equation whose kernel is

$$
\frac{\left(z^{M_{n}}-r_{1}^{M_{n}}\right)\left(z^{N_{n}}-r_{1}^{N_{n}}\right)\left(t-\alpha_{1}^{\prime}\right) \cdots\left(t-\alpha_{M_{n}}^{\prime}\right)\left(t^{N_{n}}-1\right)}{\left(z-\alpha_{1}^{\prime}\right) \cdots\left(z-\alpha_{M_{n}}^{\prime}\right)\left(z^{N_{n}}-1\right)\left(t^{M_{n}}-r_{1}^{M_{n}}\right)\left(t^{N_{n}}-r_{1}^{N_{n}}\right)} .
$$

Choose $|t|=\sigma, 1>\sigma>r_{1}, z$ on $S$; the modulus of the $n$th root of (20) has uniformly a superior limit which is not greater than $r_{1} / \sigma$, which is less than unity, so (20) approaches zero uniformly for $z$ on $S$. Choose next $|t|=\sigma_{0}$ $<\sigma_{1} \leqq|z| \leqq r_{1}<1$; then (20) is in modulus not greater than

$$
\frac{\left(t-\alpha_{1}^{\prime}\right) \cdots\left(t-\alpha_{M_{n}}^{\prime}\right)}{\left(z-\alpha_{1}^{\prime}\right) \cdots\left(z-\alpha_{M_{n}}^{\prime}\right)} \frac{2 r_{1}^{M_{n}} \cdot 2 r_{1}^{N_{n}} \cdot 2}{\left(1-r_{1}\right)\left(r_{1}-\sigma_{0}\right) r_{1}^{M_{n}-1}\left(r_{1}-\sigma_{0}\right) r_{1}^{N_{n}-1}}
$$

which approaches zero uniformly for $z$ on $S$. Precisely as in the discussion of (13), the interpolation problem of Theorem 2 can be altered by interpolating to the function

$$
\left(z-\alpha_{1}^{\prime}\right) \cdots\left(z-\alpha_{m}^{\prime}\right) f(z)
$$

by rational functions of form

$$
\left(z-\alpha_{1}^{\prime}\right) \cdots\left(z-\alpha_{m}^{\prime}\right) r_{n}(z)
$$

this modification simply requires the suppression of the factors containing $\alpha_{1}^{\prime}, \cdots, \alpha_{m}^{\prime}$ in (20); such suppression does not alter our established fact concerning the uniform approach to zero of the kernel, for $z$ on $S$ and $t$ on $|t|=\sigma$ or $|t|=\sigma_{0}$. The conclusion of Theorem 2 follows at once.

In comparison with Theorem 1 , the special situation of Theorem 2 is simpler and deals with specific formulas for the $\alpha_{k}$ and $\beta_{k}$; thus we have been able to choose $M_{n}$ (assumed to become infinite, with $M_{n} \leqq M_{n+1} \leqq M_{n}+1$ ) as an otherwise arbitrary sequence such that $M_{n} / n \rightarrow 0$. The points of interpolation $\beta_{n k}$ have been chosen as the $M_{n}$ th roots of $r_{1}^{M_{n}}$ and the $N_{n}$ th roots of $r_{1}^{N_{n}}$; consequently some points $\beta_{n k}$ occur with multiplicity greater than unity; interpolation in multiple points involves the values not merely of $f(z)$ but the values of suitable derivatives of $f(z)$. If it is desired to avoid multiple points $\beta_{n k}$, we may choose as the set $\beta_{n 1}, \beta_{n 2}, \cdots, \beta_{n n}$ the $n$th roots of $r_{1}^{n}$, or the $M_{n}$ th roots of $r_{1}^{M_{n}}$ and the $N_{n}$ th roots of $\epsilon r_{1}^{N_{n}}$, where $\epsilon$ is transcendental with modulus unity; the discussion already given requires only trivial modifica- 
tions to obtain the conclusion analogous to that of Theorem 2 .

In Theorem 2 we have not proved the convergence at points $z$ exterior to $C_{1}$. The present method fails in that case, for with $1>|z|>r_{1},|t|=\sigma_{0}<r_{1}$, the $n$th root of (20) approaches the limit $|z| / r_{1}$, which is greater than unity, so (20) does not approach zero. Indeed, the conclusion itself that $r_{n}(z)$ converges to $f(z)$ fails for $|z|>r_{1}$, unless the hypothesis of Theorem 2 is strengthened. We demonstrate this by the choice $f(z) \equiv 1 / z, \alpha_{k}^{\prime} \neq 0$. Then we have (op. cit., p. 185)

$$
f(z)-r_{n}(z)=-\frac{\left(z^{M_{n}}-r_{1}^{M_{n}}\right)\left(z^{N_{n}}-r_{1}^{N_{n}}\right)\left(-\alpha_{1}^{\prime}\right) \cdots\left(-\alpha_{M_{n}}^{\prime}\right)(-1)}{\left(z-\alpha_{1}^{\prime}\right) \cdots\left(z-\alpha_{M_{n}}^{\prime}\right)\left(z^{N_{n}}-1\right)\left(-r_{1}^{M_{n}}\right)\left(-r_{1}^{N_{n}}\right)(-z)} .
$$

For $1>|z|>r_{1}$ the $n$th root of the modulus of the second member of (21) behaves like

$$
|z| \cdot\left|\alpha_{1}^{\prime} \alpha_{2}^{\prime} \cdots \alpha_{M_{n}}^{\prime}\right| 1 / n / r_{1} .
$$

Unless the $\alpha_{k}$ and $M_{n}$ are subject to further restrictions, we cannot conclude that (22) is less than or equal to unity; for instance if we choose $\alpha_{1}^{\prime}, 0<\alpha_{1}^{\prime}$ $<r_{1}$, and $\alpha_{k}^{\prime}=\alpha_{1}^{\prime} / k$, we have

$$
\left|\alpha_{1}^{\prime} \alpha_{2}^{\prime} \cdots \alpha_{M_{n}}^{\prime}\right|^{1 / n}>\left(\alpha_{1}^{\prime} / M_{n}\right)^{M_{n} / n}
$$

yet we also have

$$
\log \left(\frac{\alpha_{1}^{\prime}}{M_{n}}\right)^{M_{n} / n}=\frac{M_{n}}{n}\left(\log \alpha_{1}^{\prime}-\log M_{n}\right),
$$

which with the choice $M_{n} \leqq n^{1 / 2}$ approaches zero; then (22) is greater than unity for $n$ sufficiently large and the second member of (21) does not converge.

The method used in Theorem 1 consists in using the two regions, $A$ and the interior of $C_{1}$, in defining points distributed on the boundaries of these regions uniformly with respect to suitable parameters, and then mixing the points so as to obtain new and useful sequences $\alpha_{k}$ and $\beta_{k}$. The method of mixing uniformly distributed points in various proportions has been used previously (op. cit., p. 215), but in both Theorems 1 and 2 the relative proportion of points $\alpha_{k}^{\prime}$ in the set $\alpha_{1}, \alpha_{2}, \cdots, \alpha_{n}$ or $\alpha_{n 1}, \alpha_{n 2}, \cdots, \alpha_{n n}$ as measured by $\lim _{n \rightarrow \infty} M_{n} / n$ is zero, a novel situation in the theory of interpolation. Because of this unusual choice, we have been able to prove convergence of the sequence $r_{n}(z)$ to $f(z)$ at every point interior to $C_{1}$ at which $f(z)$ is analytic; such convergence cannot be proved if the relative proportion of the points $\alpha_{k}^{\prime}$ is positive. As a price for this generality we have relinquished convergence exterior to $C_{1}$.

The effective use of points that are mixed in the relative proportions zero 
and unity is not limited to a region which is a punctured simply connected region:

TheOREM 3. Let $C_{0}, C_{1}$, and $C_{2}$ be the respective circles $|z|=1,|z|=r_{1}$, $|z|=r_{2}$, with $1>r_{1}>r_{2}>0$. Let $\left\{M_{n}\right\}$ be a monotonic increasing unbounded sequence of integers with $M_{n+1} \leqq M_{n}+1 \leqq n$ and $\lim _{n \rightarrow \infty} M_{n} / n=0$; we set $N_{n}=n$ $-M_{n}$. For each $n$ let the points $\alpha_{n 1}, \alpha_{n 2}, \cdots, \alpha_{n n}$ consist of the $M_{n}$ th roots of $r_{2}^{M_{n}}$ and the $N_{n}$ th roots of unity, and let the points $\beta_{n 1}, \beta_{n 2}, \cdots, \beta_{n n}$ consist of the $M_{n}$ th roots of $r_{1}^{M_{n}}$ and the $N_{n}$ th roots of $r_{1}^{N_{n}}$. Let $r_{n}(z)$ denote the rational function of form (19) defined by interpolation to $f(z)$ in the points $\beta_{n k}$, where $f(z)$ is an arbitrary function analytic on the set $\left(r_{2} \leqq\right) r_{3}<|z| \leqq r_{1}$. Then the sequence $r_{n}(z)$ converges to $f(z)$ on the set $r_{3}<|z| \leqq r_{1}$, uniformly on any closed subset.

In the usual interpolation formula the kernel now becomes

$$
\frac{\left(z^{M_{n}}-r_{1}^{M_{n}}\right)\left(z^{N_{n}}-r_{1}^{N_{n}}\right)\left(t^{M_{n}}-r_{2}^{M_{n}}\right)\left(t^{N_{n}}-1\right)}{\left(z^{M_{n}}-r_{2}^{M_{n}}\right)\left(z^{N_{n}}-1\right)\left(t^{M_{n}}-r_{1}^{M_{n}}\right)\left(t^{N_{n}}-r_{1}^{N_{n}}\right)} .
$$

Let $S$ be the set $\left(r_{2} \leqq r_{3}<\right) \rho \leqq|z| \leqq r_{1}$, and let $\Gamma$ and $\gamma$ be respectively $|t|=\rho_{1}$, $r_{1}<\rho_{1}<1$, and $|t|=\rho_{2}, r_{3}<\rho_{2}<\rho$, where we suppose $f(z)$ analytic, $r_{3}<|z| \leqq \rho_{1}$. For $t$ on $\Gamma$ and $z$ on $S$ the modulus of the maximum of the $n$th root of (23) has a superior limit which is not greater than $r_{1} / \rho_{1}$, which is less than unity, so (23) approaches zero uniformly for $t$ on $\Gamma$ and $z$ on $S$. For $t$ on $\gamma$ and $z$ on $S$ the modulus of (20) is not greater than

$$
\frac{2 r_{1}^{M_{n}} \cdot 2 r_{1}^{N_{n}} \cdot 2 \rho_{2}^{M_{n}} \cdot 2}{\left(\rho_{1}-r_{2}\right) \rho_{1}^{M_{n}-1} \cdot\left(1-r_{1}\right) \cdot\left(r_{1}-\rho_{2}\right) r_{1}^{M_{n}-1} \cdot\left(r_{1}-\rho_{2}\right) r_{1}^{N_{n}-1}},
$$

which approaches zero uniformly. Theorem 3 now follows at once.

Theorems $1-3$ remain valid in the limiting cases, where $C_{0}$ and $C_{2}$ are replaced by the points $\infty$ and 0 respectively.

HaRvard University,

Cambridge. Mass. 